Pierre Drolet MD FRCPC, Michel Girard MD MHPE FRCPC

\title{
L'utilisation du sulfate de magnésium pendant la chirurgie du phéochromocytome: à propos de deux cas
}

Le contrôle de l'hémodynamie durant l'anesthésie pour la résection d'un phéochromocytome demeure un défi pour les anesthésistes. Si l'utilisation du sulfate de magnésium est généralement bien acceptée pour le traitement de l'hypertension dans la prééclampsie, son usage pendant la résection du phéochromocytome n'a été rapporté que par un seul auteur. Nous décrivons son utilisation comme principal moyen de contrôler la pression artérielle lors de la résection d'un phéochromocytome chez deux patients. Les mécanismes de son action hypotensive, antiarythmique et anti-adrénergique sont revus. Des doses totales de 11 et 12 grammes ont été administrées aux deux patients sous forme de bolus et perfusion associée. Le sulfate de magnésium pourrait faire partie de l'arsenal thérapeutique utilisé par l'anesthésiste pendant la résection d'un phéochromocytome.

Maintenance of haemodynamic stability during anaesthesia for phaeochromocytoma resection is still a challenge. If magnesium sulfate is widely used for the control of arterial pressure during preeclampsia, its use during phaeochromocytoma resection has only been published by one author. We describe two cases where magnesium sulfate is the main agent used to control arterial pressure during resection of a phaeochromocytoma. Magnesium sulfate's hypotensive, antiarrhythmic and antiadrenergic properties are reviewed. The total doses administered were $11 \mathrm{~g}$ and $12 \mathrm{~g}$, given as an infusion and boluses. Magnesium sulfate could be part of the anaesthetist's pharmacopoeia during phaeochromocytoma resection.

\section{Key words}

IONS: magnesium;

SURGERY: phaeochromocytoma;

COMPLICATIONS: hypertension.

Département d'anesthésie-réanimation, Hôpital MaisonneuveRosemont et Université de Montréal, Montréal, Québec.

Adresser la correspondance $a$ : Dr Pierre Drolet,

Département d'anesthésie-réanimation, Hôpital Maisonneuve-

Rosemont, 5415 boul. Rosemont, Montréal, Québec

H.1T $2 \mathrm{M} 4$.

Accepté pour publication le 26 février, 1993.
Bien que l'utilisation du sulfate de magnésium $\left(\mathrm{MgSO}_{4}\right)$ pour le contrôle de la pression artérielle lors de la prééclampsie soit reconnue, ${ }^{1,2}$ son usage pour le contrôle hémodynamique pendant l'anesthésie chez le porteur d'un phéochromocytome n'a été rapporté que par James et coll. ${ }^{3-5}$ Nous présentons ici notre expérience avec deux malades dont un se distingue par des taux exceptionnellement élevés de catécholamines. Nous discutons aussi des particularités de cette technique.

\section{Observations}

\section{Patiente A}

Il s'agit d'une patiente de 37 ans $(56 \mathrm{~kg}$ ) qui consulte à plusieurs reprises au cours des neuf dernières années. Elle se plaint de troubles visuels, de palpitations et de douleurs rétrosternales accompagnées de crises hypertensives. Elle est admise à l'hôpital avec un diagnostic présumé de phéochromocytome, confirmé par des dosages de catécholamines urinaires et plasmatiques (Tableau I) et une tomodensitométrie qui met en évidence une masse de $8,5 \times 7 \mathrm{~cm}$ au niveau de la glande surrénale gauche. Elle reçoit phénoxybenzamine $10 \mathrm{mg}$ deux fois par jour per os et est conduite en salle d'opération neuf jours plus tard, lorsque la pression artérielle est jugée stable. Le matin de la chirurgie, on lui administre phénoxybenzamine $10 \mathrm{mg}$ sans autre médication. En plus du monitorage de base (ECG, saturomètre, capnographe, neurostimulateur), trois cathéters sont installés: pour l'apport liquidien dans une veine périphérique, pour le monitorage de la tension veineuse centrale (TVC) dans la jugulaire interne droite et dans l'artère radiale gauche pour la mesure de la tension artérielle. L'induction de l'anesthésie est réalisée avec midazolam $8 \mathrm{mg}$, alfentanil $5 \mathrm{mg}$ et vécuronium $7 \mathrm{mg}$. L'anesthésie est maintenue avec lisoflurane $(0,7$ à $1,7 \%)$ dans l'air et l'oxygène et un bolus d'alfentanil $2,5 \mathrm{mg}$ une heure après l'induction. Le contrôle de la pression artérielle pendant l'opération est assuré par une perfusion de $\mathrm{MgSO}_{4}$ (Figure 1).

On supplémente avec des bolus de $\mathrm{MgSO}_{4}$ au moment 
TABLEAU I Mesures préopératoires de catécholamines

\begin{tabular}{lccc}
\hline & Normales & Patient A & Patient B \\
\hline Adrénaline urinaire & $0-110 \mathrm{nmol} \cdot \mathrm{d}^{-1}$ & 437 & 3135 \\
Noradrénaline urinaire & $70-475 \mathrm{nmol} \cdot \mathrm{d}^{-1}$ & 624 & 17600 \\
Adrénaline plasmatique & $55-820 \mathrm{pmol} \cdot \mathrm{L}^{-1}$ & 4125 & 23300 \\
Noradrénaline plasmatique & $1,180-3,550 \mathrm{mmol} \cdot \mathrm{L}^{-1}$ & 6133 & 120860 \\
\hline
\end{tabular}

TABLEAU Il Mesures peropératoires de catécholamines plasmatiques $\left(\mathrm{pmol} \cdot \mathrm{L}^{-1}\right.$ )

\begin{tabular}{|c|c|c|c|c|}
\hline \multirow[b]{2}{*}{ Prélèvements* } & \multicolumn{2}{|l|}{ Patient $A$} & \multicolumn{2}{|l|}{ Patient B } \\
\hline & Adrénaline & Noradrénaline & Adrénaline & Noradrénaline \\
\hline $\mathrm{cl}$ & 3780 & 3770 & 8370 & 15490 \\
\hline$c 2$ & 4140 & 3640 & 2480 & 11990 \\
\hline c3 & 180700 & 337850 & 15830 & 91350 \\
\hline 04 & 17655 & 34170 & 103600 & 757400 \\
\hline c5 & & & 312400 & 1070500 \\
\hline$c 6$ & & & 13075 & 38560 \\
\hline
\end{tabular}

*Pour la chronologie des prélèvements $\mathrm{cl}$ à $c 6$, voir les Figures $l$ et 2 .

TABLEAU III Mesures per et postopératoires de la magnésémie $\left(\mathrm{mmol}^{-1}\right)$

\begin{tabular}{lll}
\hline Prélèvements & Patient $A$ & Patient $B$ \\
\hline Préop. & 0,71 & 0,85 \\
mI* & 1,79 & 1,5 \\
m2* $^{*}$ & 1,63 & 1,83 \\
m3* & 1,96 & 1,86 \\
S.de réveil 1 & & 1,45 \\
S.de réveil 2 & & 1,42 \\
\hline
\end{tabular}

*Pour la chronologie des prélèvements, voir les Figures 1 et 2.

de l'induction et pendant la manipulation de la tumeur. Des dosages plasmatiques de catécholamines (Tableau II) et de magnésium (Tableau III) sont faits aux moments critiques (Figure 1). Le contrôle hémodynamique est jugé très satisfaisant, ne nécessitant que deux bolus de propanolol $1 \mathrm{mg}$ pendant la chirurgie au moment d'épisodes de tachycardie sinusale. Les myorelaxants sont renversés (glycopyrrolate $0,6 \mathrm{mg}$ et néostigmine $3,0 \mathrm{mg}$ ) à la fin de l'opération et la patiente est extubée 15 minutes plus tard. On lui administre de l'albumine $25 \%(100 \mathrm{ml})$ à la salle de réveil pour maintenir une pression artérielle systolique de l'ordre de 90 à $100 \mathrm{mmHg}$. Les examens pathologiques et immuno-histochimiques de la masse confirment qu'il s'agit d'un phéochromocytome.

\section{Patient $B$}

Un homme âgé de 26 ans $(67 \mathrm{~kg})$ consulte pour une perte de vision à l'oeil gauche. L'ophtalmologiste constate une rétinopathie hypertensive grave. L'investigation

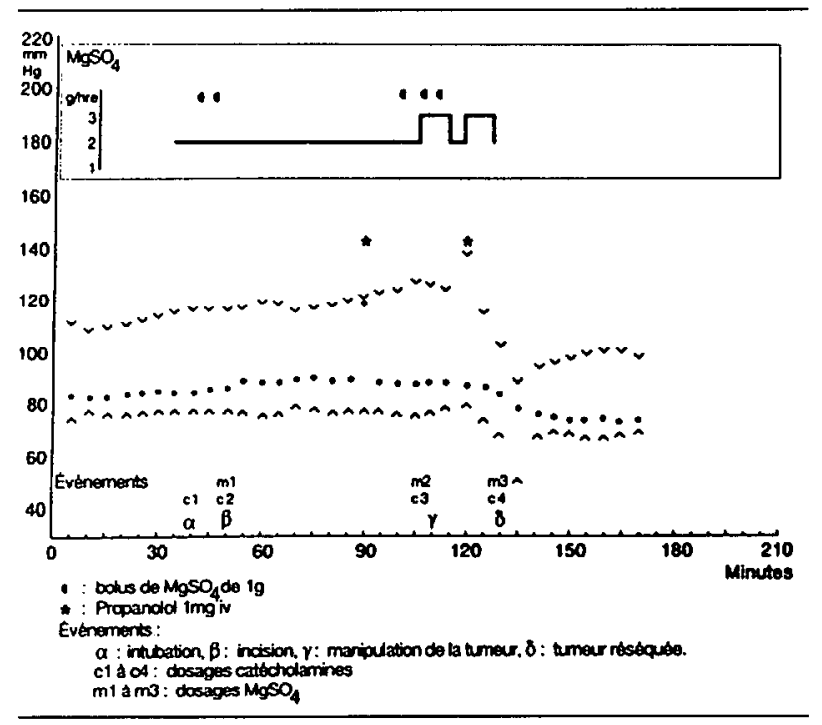

FIGURE 1 Signes vitaux de la patiente A.

néphrologique confirme la présence d'un phéochromocytome. Les dosages des catécholamines urinaires et plasmatiques préopératoires sont très élevés (Tableau I). La magnésémie préopératoire est de $0,85 \mathrm{mmol} \cdot \mathrm{L}^{-1}$ (normale: $0,75-0,95$ ). Une scintigraphie rénale de localisation au glucoheptolate montre une intense captation au pôle supérieur du rein droit sans captation myocardique. $\AA$ l'échographie, on localise une tumeur de $7,5 \mathrm{~cm}$ de diamètre au pôle supérieur du rein droit.

Avant le début du traitement médical, on note des pressions artérielles qui atteignent $210 / 170$. Un traitement per 
TABLEAU IV Mesures hémodynamiques peropératoires du patient B

\begin{tabular}{lcccc}
\hline Minutes après l'arrivée en s.d'op. & 100 & 115 & 145 & \multicolumn{1}{l}{180} \\
\hline Débit cardiaque $\left(\mathrm{L} \cdot \mathrm{min}^{-1}\right)$ & 3,44 & 2,56 & 2,92 & 2,58 \\
Fréquence cardiaque $\left(\mathrm{b} \cdot \mathrm{min}^{-1}\right)$ & 70 & 70 & & 68 \\
Pression capillaire bloquée $(\mathrm{mmHg})$ & 27 & 27 & & 16 \\
Pression systolique $(\mathrm{mmHg})$ & 93 & 93 & & 110 \\
Pression diastolique $(\mathrm{mmHg})$ & 63 & 63 & & 66 \\
Résistance vasculaire systémique & & & & 1972 \\
(dynes $\cdot \mathrm{s} \cdot \mathrm{cm}^{-5}$ ) & 1276 & 1713 & & \\
\hline
\end{tabular}

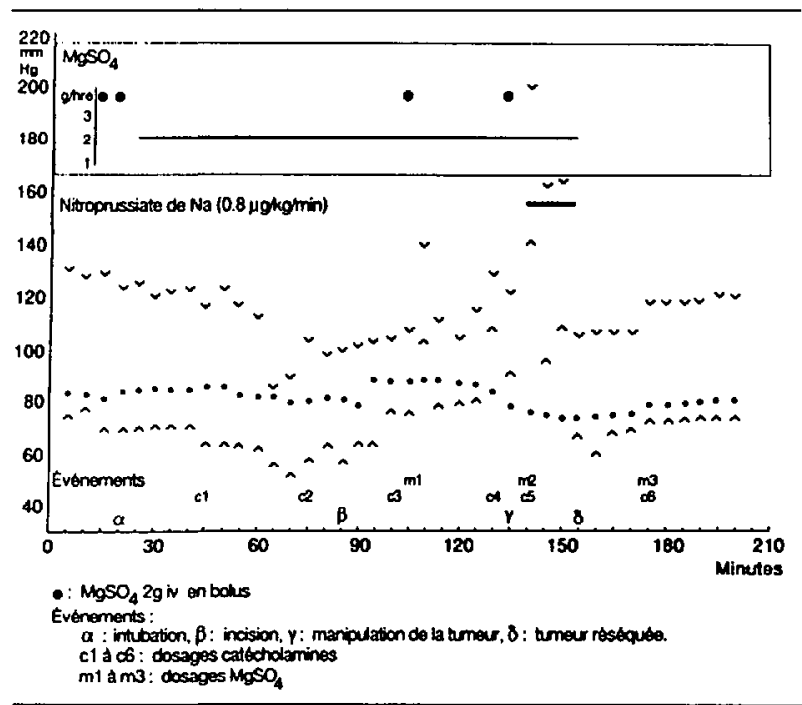

FIGURE 2 Signes vitaux du patient $B$.

os est entrepris avec de l'énalapril $5 \mathrm{mg}$ deux fois par jour et de la prazosine $2 \mathrm{mg}$ deux fois par jour. Par la suite, on remplace par de la phénoxybenzamine 20 mg deux fois par jour et de l'aténolol, $100 \mathrm{mg}$ par jour.

La prémédication consiste en du lorazépam $2 \mathrm{mg}$, de la morphine $6 \mathrm{mg}$ et de la scopolamine $0,4 \mathrm{mg}$. À l'arrivée en salle d'opération, un bolus de $\mathrm{MgSO}_{4}$ de $4 \mathrm{~g}$ est administré en 20 minutes, par la suite une perfusion de $2 \mathrm{~g} \cdot \mathrm{h}^{-1}$ est mise en marche; 2 bolus de $2 \mathrm{~g}$ seront administrés durant la chirurgie (Figure 2). Le même monitorage que pour la patiente $\mathrm{A}$ est utilisé, à l'exception d'un cathéter de Swan-Ganz au lieu d'un cathéter de mesure de la TVC.

Le malade reçoit à l'induction propanolol $3,2 \mathrm{mg}$, fentanyl $0,30 \mathrm{mg}$, alfentanil $2,5 \mathrm{mg}$, midazolam $10 \mathrm{mg}$ et vécuronium $20 \mathrm{mg}$. Des dosages plasmatiques de catécholamines ont été faits à six reprises (Figure 2) et se retrouvent au Tableau II. L'évolution des signes vitaux se trouve à la Figure 2. Nous avons dosé le $\mathrm{MgSO}_{4}$ (Tableau III) à cinq reprises durant lintervention (Figure 2) et deux fois à la salle de réveil. Nous avons dû perfuser du nitroprussiate durant 15 minutes, pour une dose totale de $750 \mu \mathrm{g}$, au moment de la manipulation de la tumeur par le chirurgien.

Nous avons maintenu l'anesthésie avec un mélange $\mathrm{N}_{2} \mathrm{O} / \mathrm{O}_{2}$, du fentanyl $(0,2 \mathrm{mg})$ et du vécuronium $(10 \mathrm{mg})$ et administré de l'isoflurane (de 0 à $1,7 \%$ ) au besoin. Plusieurs données hémodynamiques peropératoires sont rapportées au Tableau IV, témoignant d'une pression artérielle normale, de résistances vasculaires périphériques élevées et d'un débit cardiaque marginal. Les myorelaxants sont renversés (néostigmine $2,5 \mathrm{mg}$ et glycopyrrolate $0,5 \mathrm{mg}$ ) à la salle de réveil.

L'analyse des prélèvements urinaires et sanguins de catécholamines des deux patients a été faite par chromatographie en phase liquide avec un détecteur électrochimique (Waters, Division de Chromatographie, 3688 Nashua Drive, Mississauga, Ontario, L4N IM5).

\section{Discussion}

L'usage de $\mathrm{MgSO}_{4}$ pour la chirurgie du phéochromocytome a été rapporté pour la première fois en $1985 .^{3}$ L'auteur suggère alors que l'activité pharmacologique du $\mathrm{MgSO}_{4}$ sur la libération et l'action périphérique des catécholamines en fait un médicament de choix pour diminuer les effets hémodynamiques qu'elles provoquent. En effet, plusieurs facteurs militent en faveur de l'utilisation du $\mathrm{MgSO}_{4}$ pour le traitement et la prévention des états hyper-adrénergiques. Tout d'abord, il a été démontré que le $\mathrm{MgSO}_{4}$ peut atténuer la libération de catécholamines tant au niveau des terminaisons sympathiques que de la médullo-surrénale. ${ }^{6-9}$ Cependant, cet effet inhibiteur sur la médullo-surrénale est peu significatif au moment de la manipulation tumorale et du stimulus mécanique. ${ }^{5,6,10}$ Deuxièmement, le $\mathrm{MgSO}_{4}$ peut se comporter comme un vasodilatateur puissant. ${ }^{11,12} \mathrm{On}$ attribue son activité à un effet direct sur les vaisseaux ${ }^{13}$ associé à son antagonisme de vasoconstricteurs connus comme l'épinéphrine. ${ }^{14}$ Troisièmement, bien que des études in vivo attribuent un effet inotrope négatif au $\mathrm{MgSO}_{4}{ }^{15,16}$ les essais cliniques sont moins catégoriques, les indices de performance cardiaque n'étant pas modifiés de façon significative. ${ }^{17-24}$ En revanche, il se révèle comme 
un excellent anti-arythmique sur un modèle expérimental d'arythmies provoquées par l'adrénaline chez le rat ${ }^{25}$ et le babouin. ${ }^{26}$ Une dose initiale de 40 à $60 \mathrm{mg} \cdot \mathrm{kg}^{-1}$, suivie d'une perfusion de $2 \mathrm{~g} \cdot \mathrm{h}^{-1}$ et supplémentée par des bolus de 1 à $3 \mathrm{~g}$ au moment de lintubation et des manipulations tumorales, semble adéquate. ${ }^{5,10}$ Une magnésémie basse au départ peut nécessiter des doses plus fortes. ${ }^{4}$

Le $\mathrm{MgSO}_{4}$ a aussi d'autres conséquences sur la conduite de l'anesthésie. Son inhibition du système nerveux s'exerce aussi au niveau de la jonction neuromusculaire. ${ }^{27}$ Il peut donc prolonger l'effet des myorelaxants non dépolarisants ${ }^{28,30}$ et peut-être celui des dépolarisants. ${ }^{29,31-33}$ On suggère donc l'utilisation de médicaments de durée intermédiare comme le vécuronium. Dans le cas de bloc neuromusculaire excessif, on peut administrer du gluconate de calcium mais ceci semble inutile. En effet, chez 12 malades sous alcuronium une magnésémie moyenne de $1,9 \mathrm{mmol} \cdot \mathrm{L}^{-1}$ n'a pas causé de problème d'antagonisme. ${ }^{5} \mathrm{La}$ période post-résection peut s'accompagner d'hypotension artérielle. Celle-ci répond généralement bien au remplissage vasculaire. ${ }^{34,35}$ Si elle persiste, le gluconate de calcium peut être employé pour renverser les effets du $\mathrm{MgSO}_{4}{ }^{6}{ }^{6} \mathrm{Ce}$ dernier s'élimine cependant rapidement par voie rénale dans les heures qui suivent l'arrêt de la perfusion. ${ }^{36,37}$

Les cas rapportés ici sont deux exemples de phéochromocytomes très actifs, particulièrement celui du patient B. En effet, lorsque nous comparons ces patients à ceux de James, ${ }^{4,5}$ nous notons que dans le cas de quatre malades sur lesquels les dosages préopératoires de catécholamines sont effectués, les taux plasmatiques maximaux de noradrénaline et d'adrénaline sont respectivement de $40,000 \mathrm{pmol} \cdot \mathrm{L}^{-1}$ et de $2,100 \mathrm{pmol} \cdot \mathrm{L}^{-1}$. De plus, James rapporte des taux peropératoires ne dépassant jamais $84,000 \mathrm{pmol} \cdot \mathrm{L}^{-1}$ de noradrénaline et $2,300 \mathrm{pmol} \cdot \mathrm{L}^{-1}$ d'adrénaline. Nous croyons donc que les observations présentées ici, particulièrement le patient $B$ pour qui les taux de catécholamines préopératoires et au moment de la manipulation tumorale sont de plusieurs fois supérieurs (Tableau I et II), indiquent que l'usage du $\mathrm{MgSO}_{4}$ peut s'avérer utile même dans le cas de tumeurs très actives sur le plan métabolique. Nous constatons de plus que l'usage à faible dose de nitroprussiate de sodium n'est devenu nécessaire que chez le patient $B$ et cela pour une courte période (Figure 2). Cette situation s'explique par l'importante activité métabolique de la tumeur combinée à la stimulation mécanique de la résection. C'est d'ailleurs le moment où il faut être le plus vigilant, le $\mathrm{MgSO}_{4}$ étant moins efficace pendant la manipulation directe de la tumeur. Les doses totales de $\mathrm{MgSO}_{4}$ utilisées sont de 11 $\mathrm{g}$ dans le cas du patient $\mathrm{A}$ et $12 \mathrm{~g}$ dans le cas du patient B. Il s'agit là de doses conservatrices comme en témoigne la magnésémie sérique qui varie dans les deux cas entre
1,5 et $1,9 \mathrm{mmol} \cdot \mathrm{L}^{-1}$ (Tableau III). James ${ }^{5}$ rapporte en effet des taux moyens de $1,9 \mathrm{mmol} \cdot \mathrm{L}^{-1}$ et des taux maximaux de $4 \mathrm{mmol} \cdot \mathrm{L}^{-1}$. Bien que l'effet des myorelaxants soit prolongé par le $\mathrm{MgSO}_{4}$, le vécuronium a facilement été renversé à la salle de réveil dans les deux cas. Les mesures hémodynamiques rapportées au Tableau IV témoignent chez le patient $B$ d'une réserve cardiaque diminuée ce qui est toujours une possibilité chez le porteur de phéochromocytome. ${ }^{38,39}$ On attribue cet état à une exposition excessive du myocarde aux catécholamines. ${ }^{38-43}$

En conclusion, ces deux observations suggèrent que le sulfate de magnésium pourrait jouer un rôle important sur le contrôle de la pression durant l'anesthésie des patients porteurs de phéochromocytomes. Il peut être associé aux méthodes conventionnelles d'anesthésie et de maintien de la pression artérielle sans inconvénients majeurs.

\section{Remerciements}

Les auteurs désirent remercier M. David Geadah, Ph.D. pour les mesures de catécholamines urinaires et plasmatiques, de même que Mesdames Christiane Côté B.Sc.inf., Sylvie Le Breux et le Dr Yves Veillette pour la préparation et la révision du manuscrit.

\section{Références}

1 Pritchard JA, Pritchard SA. Standardized treatment of 154 consecutive cases of eclampsia. Am J Obstet Gynecol 1975; 123: 543-52.

2 Pritchard JA, Cunningham G, Pritchard SA. The Parkland Memorial Hospital protocol for treatment of eclampsia: evaluation of 245 cases. Am J Obstet Gynecol 1978; 148: 951-63.

3 James MFM. The use of magnesium sulfate in the anesthetic management of pheochromocytoma. Anesthesiology 1985; 62: 188-90.

4 James MFM, Huddle KRL, Owen $A D$, van der Veen $B W$. Use of magnesium sulfate in the anaesthetic management of phaeochromocytoma in pregnancy. Can J Anaesth 1988; 35: 178-82.

5 James MFM. Use of magnesium sulfate in the anaesthetic management of phaeochromocytoma: a review of 17 anaesthetics. Br J Anaesth 1989; 62: 616-23.

6 Douglas $W W$, Rubin RP. The mechanism of cathecholamine release from the adrenal medulla and the role of calcium in stimulus-secretion coupling. J Physiol (London) 1963; 167: 288-310.

7 Von Euler US, Lishajko F. Effects of $\mathrm{Mg}^{++}$and $\mathrm{CA}^{++}$ on noradrenaline release and uptake in adrenergic nerve granules in different media. Acta Physiol Scand 1973; 89: 415-522.

8 Kirpekar SM, Misu Y. Release of noradrenaline by splenic nerve stimulation and its dependence on calcium. J Physiol 1967; 188: 219-34. 
9 Nordes JP, Wacker WEC. Excess magnesium. Pharmacol Rev 1978; 29: 273-300.

10 James MFM. Clinical use of magnesium infusions in anesthesia. Anesth Analg 1992; 74: 129-36.

11 Altura BM, Altura BT. Role of magnesium ions in contractility of blood vessels and skeletal muscles. Magnesium Bulletin 1982; 1a: 102-14.

12 Altura BM, Altura BT. Magnesium ions and contraction of vascular smooth muscle in relationship to some vascular diseases. Fed Proc 1981; 40: 2674-9.

13 Altura BM, Altura BT. Magnesium and vascular tone and reactivity. Blood Vessels 1978; 15: 5-16.

14 Altura $B M . \mathrm{Mg}^{2+}-\mathrm{CA}^{2+}$ interaction in contractility of vascular smooth muscle: $\mathrm{Mg}^{2+}$ versus organic calcium channel blockers on myogenic tone and agonist-induced responsiveness of blood vessels. Can J Physiol Pharmacol 1987; 65: 729-45.

15 Shine KI. Myocardial effects of magnesium. Am J Physiol 1979; 237: H413-23.

16 Paddle $B M$, Haugaard $N$. Role of magnesium in effects of epinephrine on heart contraction and metabolism. Am J Physiol 1971; 221: 1178-84.

17 Bristow MR, Daniels RS, Kernoff RS, Harrison DC. Effect of D600, practolol, and alterations in magnesium on ionized calcium concentration-response relationships in the intact dog. Circ Res 1977; 41: 574-81.

18 Charbon GA. Unloading the heart by magnesium: the natural calcium competitor. Magnesium 1983; 2: 36-45.

19 Aldrete JA, Barnes DR, Aikawa JK. Does magnesium produce anesthesia? Anesth Analg 1968; 47: 428-33.

20 Critelli G, Ferro G, Peschle C, Perticone FR, Rengo FR, Condoreli $M$. Myocardial contractility after injection or prolonged infusion of magnesium sulfate. Acta Cardiol $1977 ; 32: 65-73$.

21 Wallis DE, Gierke LW, Scanlon PJ, Wolfson PM, Kopp SJ. Sustained postischemic cardiodepression following magnesium-diltiazem cardioplegia. Proc Soc Exp Biol Med 1986; 182: 375-85.

22 Dandavino A, Woods JR, Murayamak K, Brinkman C, Assali NS. Circulatory effects of magnesium sulfate in normotensive and renal hypertensive pregnant sheep. Am J Obstet Gynecol 1977; 127: 759-74.

23 James MFM, Cork RC, Dennett JE. Cardiovascular effects of magnesium sulfate in the baboon. Magnesium 1987; 6: 314-24.

24 Cotton DB, Gonik B, Karen F, Dornan RN.

Cardiovascular alterations in severe pregnancy-induced hypertension: acute effects of intravenous magnesium sulfate. Am J Obstet Gynecol 1984; 148: 162-5.

25 Mayer DB, Miletich DJ, Feld JM, Albrecht RF. The effects of magnesium salts on the duration of epinephrineinduced ventricular tachyarrhythmias in anesthetized rats. Anesthesiology 1989; 71: 923-8.
26 James MFM, Cork RC, Harlen GM, White JF. Interactions of adrenaline and magnesium on the cardiovascular system of the baboon. Magnesium 1988; 7: 37-43.

27 Krendal $D A$. Hypermagnesemia and neuromuscular transmission. Semin Neurol 1990; 10: 42-5.

28 Ghonheim MM, Long JP. The interaction between magnesium and other neuromuscular blocking agents. Anesthesiology 1970; 32: 23-7.

29 Giesecke AH, Morris RE, Dalton MD, Stephen CR. Of magnesium, muscle relaxants, toxic parturients and cats. Anesth Analg 1968; 47: 689-93.

30 Sinatra RS, Philip BK, Naulty JS, Ostheimer GW. Prolonged neuromuscular blockade with vecuronium in a patient treated with magnesium sulfate. Anesth Analg 1985; 64: 1220-2.

31 Skaredoff $M N$, Roaf ER, Datta $S$. Hypermagnesaemia and anaesthetic management. Can Anaesth Soc J 1982; 29: 35-41.

32 James MFM, Cork RC, Denett JE. Succinylcholine pretreatment with magnesium sulfate. Anesth Analg 1986; 65: 373-6.

33 Baraka A, Yazigi A. Neuromuscular interaction of magnesium with succinylcholine-vecuronium sequence in the eclamptic parturient. Anesthesiology 1987; 67: 806-8.

34 Katz J, Benumof J, Kadis LB. Anesthesia and Uncommon Diseases. 2nd ed. Philadelphia: W.B. Saunders Co., 1981.

35 Stoelting $R K$, Dierdof $S F$, McCammon $R L$. Anesthesia and Co-Existing Disease. 2nd ed. New York: Churchill Livingstone Inc., 1988.

36 Quamme GA, Dirks JH. The physiology of renal magnesium handling. Renal Physiol 1986; 9: 257-69.

37 Smith WO, Baxter DJ, Lindner A, Ginn HE. Effect of magnesium depletion on renal function in the rat. $\mathbf{J} \mathbf{L a b}$ Clin Med 1962; 59: 211-9.

38 Hoffman BB. Adrenergic pharmacology in rats harboring pheochromocytoma. Hypertension 1991; 18: 35-9.

39 Hicks RJ, Wood B, Kalff V, Anderson ST, Kelly MJ. Normalization of left ventricular ejection fraction following resection of pheochromocytoma in a patient with dilated cardiomyopathy. Clin Nucl Med 1991; 16: 413-6.

40 Wilkenfeld $C$, Cohen $M$, Lansman $S L$, et al. Heart transplantation for end-stage cardiomyopathy caused by an occult pheochromocytoma. J Heart Lung Transplant 1992; 11: 363-6.

41 Balazovjech I, Murin J, Kralova K. Catecholamine heart muscle disease in pheocromocytoma. Exp Clin Endocrinol 1989; 93: 29-36.

42 Scott I, Parkes R, Cameron DP. Phaechromocytoma and cardiomyopathy. Med J Aust 1988; 148: 94-6.

43 Malingue S, Dheilly $M$, Pennarguear A, Perchec F, Tanguy $L$. Hypertrophic candiomyopathy secondary to pheochromocytoma and surgical indications. Cah Anesthesiol 1988; 36: 651-3. 